\title{
High Test Reference Range
}

National Cancer Institute

\section{Source}

National Cancer Institute. High Test Reference Range. NCI Thesaurus. Code C70933.

Reported upper limit of the test reference range. 